\title{
Diabetes mellitus e hipertensión arterial como factor de riesgo de mortalidad en pacientes con Covid-19
}

\section{Diabetes mellitus and arterial hypertension as a risk factor of mortality in patients with Covid-19}

Pedro Javier Navarrete-Mejía ${ }^{1,2, a}$; Frank Antonio Lizaraso-Soto ${ }^{1, b, c}$; Juan Carlos Velasco-Guerrero ${ }^{3, \mathrm{a}, \mathrm{d}}$; Luis Melchor Loro-Chero,

\begin{abstract}
RESUMEN
Objetivo. identificar la posible asociación de diabetes mellitus e hipertensión arterial como factores de riesgo de mortalidad en pacientes con COVID-19. Material y métodos: estudio de cohorte prospectivo, incluye a 1947 pacientes con 30 o más años de edad, atenciones realizadas entre marzo y agosto del 2020; pacientes con diagnóstico clínico y laboratorial de COVID-19; se excluyeron pacientes con inconsistencias en la información registrada, fallecidos en primeras 24 horas o estuvieron muertos al ingreso. Se incluyeron variable periodo de atención, edad, sexo, diabetes mellitus, hipertensión arterial, estancia hospitalaria, frecuencia respiratoria, frecuencia cardiaca, saturación de oxígeno y temperatura; para el análisis se utilizó la prueba chi cuadrado de Pearson, Odds Ratio y prueba $T$ de student para muestras no emparejadas ( $p<0,05)$. Resultados: $73 \%$ de los fallecidos de sexo masculino ( $p<0.001)$, mayor frecuencia entre los 60 - 79 años de edad $(54,8 \%, p<0,001)$; diabetes como comorbilidad en $17 \%$ de los casos $(p=0,019)$ e hipertensión arterial en $24 \%(p<0,001)$. Las variables sexo (OR $1,5)$ y las comorbilidades diabetes $(O R 1,4)$ e hipertensión arterial $(O R 1,9)$ representan factores de riesgo de mortalidad. Conclusiones: la diabetes mellitus e hipertensión arterial, para la población en estudio, se identificaron como factor de riesgo de mortalidad frente al COVID-19.
\end{abstract}

Palabras Clave: SARS Cov-2; mortalidad hospitalaria; pandemia. Fuente: DeCS-BIREME).

\begin{abstract}
Objetive. to identify the possible association of diabetes mellitus and arterial hypertension as risk factors for mortality in patients with COVID- 19. Material and methods: prospective cohort study, includes 1947 patients aged 30 or over, care performed between March and August 2020; patients with clinical and laboratory diagnosis of COVID-19; Patients with inconsistencies in the information recorded, who died in the first 24 hours or were dead on admission, were excluded. Variable period of care, age, sex, diabetes mellitus, arterial hypertension, hospital stay, respiratory rate, heart rate, oxygen saturation and temperature were included; Pearson's chi-square test,
\end{abstract}

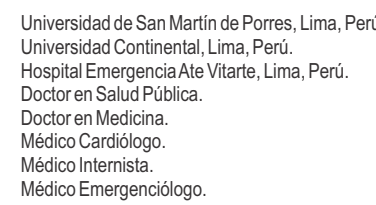

Odds Ratio and Student's T test for unpaired samples ( $\mathrm{p}$ $<0.05$ ) were used for the analysis. Results: $73 \%$ of male deaths ( $p<0.001)$, the highest frequency was between $60-79$ years of age $(54.8 \%, p<0.001)$; diabetes as comorbidity in $17 \%$ of cases $(p=0.019)$ and hypertension in $24 \%(p<0.001)$. The variables sex (OR 1.5) and the comorbidities diabetes (OR 1.4) and arterial hypertension (OR 1.9) represent risk factors for mortality. Conclusions: diabetes mellitus and arterial hypertension, for the study population, were identified as a risk factor for mortality against COVID-19

Keywords: SARS Cov-2; hospital mortality; pandemics (Source: DeCS-BIREME).

\section{INTRODUCCIÓN}

La emergencia global de Salud Pública, a causa del SARS-CoV2, nos brinda conocimiento nuevo y de alta relevancia para la lucha contra la enfermedad; hoy se tiene identificado los grupos de riesgo, pacientes con diabetes e hipertensión arterial (HTA) por ejemplo, 
estas personas desarrollan formas graves de la enfermedad. La OMS informa una tasa de letalidad media global del $2 \%$, en especial en personas del grupo de riesgo; en el Perú la tasa llega al $4.65 \%^{(1)}$.

En el caso particular de la diabetes, las infecciones virales son más difíciles de tratar, situación que se agrava en pacientes cuyos niveles de glicemia no están controlados, se conoce que en este grupo de pacientes el sistema inmune está comprometido. La HTA también es una preocupación, enfermedad que de por sí tiene complicaciones importantes para quien la padece, situación que se agrava en infección por SARS-CoV2 ${ }^{(2,3)}$.

Estudios globales reportan, en el caso particular de pacientes que llegan a las unidades de cuidados intensivos $(\mathrm{UCl})$, como comorbilidades asociadas a la letalidad la HTA $(23,7 \%)$, diabetes $(22,0 \%)$ y enfermedades coronarias $(5,8 \%)^{(4-6)}$.

La evidencia científica demuestra que el SARS-CoV2 se une a las células diana a través de la enzima convertidora de angiotensina 2 (ACE 2), expresada en células epiteliales de pulmón, intestino, riñón y vasos sanguíneos ${ }^{(4,5)}$.

En pacientes con diabetes, tratados con inhibidores de enzima convertidora de angiotensina (ECA) y bloqueadores de los receptores de la angiotensina I (ARA I), se incrementa notablemente la expresión ACE 2; la HTA también se trata con inhibidores de ECA y antagonistas de los receptores de la angiotensina (ARB). La ACE2 se incrementa con el uso de tiazolidinedionas e ibuprofeno. El incremento en la expresión ACE2 facilitaría la infección por SARS CoV2, en estos pacientes la enfermedad es grave o mortal ${ }^{(5)}$. En el Perú la prevalencia de diabetes es aproximadamente del $8 \%$ y la HTA está presente en el $28 \%$ de la población general.

Es objetivo del estudio identificar la posible asociación de diabetes mellitus e hipertensión arterial como factores de riesgo de mortalidad en pacientes con COVID-19.

\section{MATERIALY MÉTODOS}

Estudio de cohorte prospectivo, se analizaron los datos de pacientes con 30 o más años de edad atendidos en el Hospital Emergencia Ate Vitarte (HEAV), las atenciones se realizaron entre los meses de marzo y agosto del 2020; la totalidad de pacientes tenían diagnóstico clínico y de laboratorio para COVID-19, todos los casos se encuentran registrados en el Instituto Nacional de Salud del Perú. Se incluyó en el estudio 1947 pacientes, atenciones con información completa en historias clínicas electrónicas; se excluyeron pacientes con inconsistencias en la información registrada, fallecieron en las primeras 24 horas o estuvieron muertos al ingreso. La información fue proporcionada por el área de estadística del hospital.

Se incluyeron las variables periodo de atención, edad, sexo, diabetes mellitus, hipertensión arterial, estancia hospitalaria, frecuencia respiratoria, frecuencia cardiaca, saturación de oxígeno y temperatura; la información de las variables fue recabada al ingreso del paciente al hospital, no son variables de seguimiento durante la estancia; fuente de información anamnesis registrada en historia clínica del paciente.

El análisis de asociación y riesgo con la mortalidad fue analizado utilizando la prueba chi cuadrado de Pearson y Odds Ratio, los datos se estratificaron según la condición de fallecido o no; en las variables cuantitativas se determinó el intervalo de confianza y se comparó medias utilizando la prueba $T$ de student para muestras no emparejadas. Se consideró como umbral de significación estadística un valor de $p<0,05$. Todos los análisis se realizaron utilizando el SPSS v 27.

La investigación fue aprobada por la Dirección General del Hospital Emergencia Ate Vitarte y aprobada por el comité institucional de ética de la Universidad de San Martin de Porres - comité acreditado por el Instituto Nacional de Salud del Perú.

\section{RESULTADOS}

Se evidencia un incremento en el número de pacientes atendidos para el periodo de estudio, el Hospital Emergencia Ate Vitarte es un hospital nuevo que inicio la actividad asistencial a fines del mes de marzo de 2020 ; el $54,9 \%$ de los pacientes se atendieron en los meses de julio/agosto. El 60,4\% de los atendidos eran adultos y el $39,6 \%$ tenía 60 o más años; mayor frecuencia del sexo masculino $(67,1 \%)$. La diabetes e Hipertensión arterial (HTA), como comorbilidades en el total de atendidos, estuvieron presentes en el $14,4 \%$ y $17,6 \%$ de los pacientes. Se evidenció alteraciones, como media muestral, en las frecuencias respiratorias y saturación de Oxígeno; la estancia hospitalaria fue prolongada con alta variabilidad. (tabla 1 ).

El $73 \%$ de los fallecidos fueron del sexo masculino $(p<0,001)$ con mayor frecuencia etaria entre los 60 - 79 años de edad (54.8\%, $\mathrm{p}<0,001)$; este grupo de pacientes presentaban como comorbilidad la diabetes en el $17 \%$ de los casos $(p=0,019)$ e hipertensión arterial en el $24 \%$ $(p<0,001)$. Se evidenció diferencias estadísticamente significativas, entre los fallecidos y pacientes dados de alta, en las variables frecuencia cardiaca, frecuencia respiratoria, temperatura, saturación de oxígeno y estancia hospitalaria $(p<0,05)$; es necesario mencionar que los datos de estas cinco variables fueron recogidos al ingreso del paciente al hospital, no son valores de seguimiento durante el internamiento (tabla 2). 
Tabla 1. Características de la población hospitalizada por covid-19.

\begin{tabular}{|c|c|c|}
\hline Variable & $\mathrm{n}$ & $\%$ \\
\hline \multicolumn{3}{|l|}{ Fecha de atención } \\
\hline Marzo & 10 & $0,5 \%$ \\
\hline Abril & 143 & $7,3 \%$ \\
\hline Mayo & 386 & $19,8 \%$ \\
\hline Junio & 338 & $17,4 \%$ \\
\hline Julio & 557 & $28,6 \%$ \\
\hline Agosto & 513 & $26,3 \%$ \\
\hline \multicolumn{3}{|l|}{ Sexo } \\
\hline Masculino & 1306 & $67,1 \%$ \\
\hline Femenino & 641 & $32,9 \%$ \\
\hline \multicolumn{3}{|l|}{ Edad } \\
\hline $30-59$ & 1176 & $60,4 \%$ \\
\hline $60-79$ & 691 & $35,5 \%$ \\
\hline 80 a más & 80 & $4,1 \%$ \\
\hline \multicolumn{3}{|l|}{ Diabetes } \\
\hline $\mathrm{Si}$ & 280 & $14,4 \%$ \\
\hline No & 1667 & $85,6 \%$ \\
\hline \multicolumn{3}{|l|}{ Hipertensión arterial } \\
\hline $\mathrm{Si}$ & 343 & $17,6 \%$ \\
\hline No & 1604 & $82,4 \%$ \\
\hline Frecuencia cardiaca & \multicolumn{2}{|c|}{$98,95+/-17,72$ lat $/ \mathrm{min}$} \\
\hline Frecuencia Respiratoria & \multicolumn{2}{|c|}{$26,58+/-6,64 \mathrm{resp} / \mathrm{min}$} \\
\hline Temperatura & \multicolumn{2}{|c|}{$\begin{array}{c}37,09+/-0,77 \text { oC } \\
{[\text { (IC 95\%) } 37,06-37,13 \text { ] }}\end{array}$} \\
\hline Saturación Oxígeno & \multicolumn{2}{|c|}{$\begin{array}{c}85,16+/-11,66 \% \\
{[(\text { IC 95\%) } 84,64-85,67]}\end{array}$} \\
\hline Estancia hospitalaria & \multicolumn{2}{|c|}{$\begin{array}{c}9,54+/-19,03 \text { días } \\
\text { [(IC 95\%) } 8,70-10,39 \text { ] }\end{array}$} \\
\hline
\end{tabular}

La investigación determina, para la data analizada, que las variables sexo (OR 1,5), y las comorbilidades diabetes $(O R 1,4)$ e hipertensión arterial $(O R 1,9)$, son factores de riesgo de mortalidad en los pacientes mayores de 30 años atendidos, periodo marzo-agosto, en el Hospital Emergencia Ate Vitarte (tabla 3).

\section{DISCUSIÓN}

La pandemia por SARS CoV-2 está asociada con una alta morbi-mortalidad. La evidencia científica demostraría, en los pacientes con COVID-19, que las comorbilidades diabetes mellitus, hipertensión arterial (HTA), enfermedad pulmonar obstructiva crónica (EPOC) y las enfermedades cardiovasculares son las más prevalentes en asociación a la muerte del paciente. En el caso particular de la diabetes y la HTA son considerados factores de riesgo para un resultado desfavorable, sin embargo, aun la evidencia científica es contradictoria ${ }^{(6)}$.

Las limitaciones en los estudios, o contradicciones, se
Tabla 2. Características de fallecidos y altas de pacientes hospitalizados por covid-19

\begin{tabular}{|c|c|c|c|c|c|}
\hline \multirow{2}{*}{ Variable } & \multicolumn{2}{|c|}{ Fallecidos } & \multicolumn{2}{|c|}{ Altas } & \multirow{2}{*}{$\mathrm{p}$ valor } \\
\hline & $n$ & $\%$ & $\mathrm{n}$ & $\%$ & \\
\hline \multicolumn{6}{|l|}{$\begin{array}{l}\text { Fecha de } \\
\text { atención }\end{array}$} \\
\hline Marzo & 7 & $1,1 \%$ & 3 & $0,2 \%$ & \multirow{6}{*}{-- } \\
\hline Abril & 29 & $4,4 \%$ & 114 & $8,8 \%$ & \\
\hline Mayo & 158 & $24,2 \%$ & 228 & $17,6 \%$ & \\
\hline Junio & 149 & $22,8 \%$ & 189 & $14,6 \%$ & \\
\hline Julio & 174 & $26,6 \%$ & 383 & $29,6 \%$ & \\
\hline Agosto & 136 & $20,8 \%$ & 377 & $29,1 \%$ & \\
\hline \multicolumn{6}{|l|}{ Sexo } \\
\hline Masculino & 477 & $73.00 \%$ & 829 & $64,1 \%$ & \multirow{2}{*}{$<0,001^{*}$} \\
\hline Femenino & 176 & $27.00 \%$ & 465 & $35,9 \%$ & \\
\hline \multicolumn{3}{|l|}{ Edad } & & , & \\
\hline $30-59$ & 246 & $37,7 \%$ & 930 & $71,9 \%$ & \multirow{3}{*}{$<0,001^{*}$} \\
\hline $60-79$ & 358 & $54,8 \%$ & 333 & $25,7 \%$ & \\
\hline 80 a más & 49 & $7,5 \%$ & 31 & $2,4 \%$ & \\
\hline \multicolumn{6}{|l|}{ Diabetes } \\
\hline $\mathrm{Si}$ & 111 & $17.00 \%$ & 169 & $13,1 \%$ & \multirow{2}{*}{$0,019^{*}$} \\
\hline No & 542 & $83.00 \%$ & 1125 & $86,9 \%$ & \\
\hline \multicolumn{6}{|l|}{ Hipertensión } \\
\hline $\mathrm{Si}$ & 157 & $24.00 \%$ & 186 & $14,4 \%$ & \multirow{2}{*}{$<0,001^{*}$} \\
\hline No & 496 & $76.00 \%$ & 1108 & $85,6 \%$ & \\
\hline $\begin{array}{l}\text { Frecuencia } \\
\text { cardiaca }\end{array}$ & \multicolumn{2}{|c|}{$\begin{array}{l}103,37+/-18,32 \text { lat } / \mathrm{min} \\
{[(\text { IC } 95 \%) 101,97-104,78]}\end{array}$} & \multicolumn{2}{|c|}{$\begin{array}{l}96,71+/-16,99 \text { lat } / \mathrm{min} \\
{[(\mathrm{IC} 95 \%) 95,79-97,64]}\end{array}$} & $<0,001^{* *}$ \\
\hline $\begin{array}{l}\text { Frecuencia } \\
\text { Respiratoria }\end{array}$ & \multicolumn{2}{|c|}{$\begin{array}{l}29,33+/-7,04 \mathrm{resp} / \mathrm{min} \\
{[(\mathrm{IC} 95 \%) 28,79-29,87]}\end{array}$} & \multicolumn{2}{|c|}{$\begin{array}{l}25,19+/-5,97 \mathrm{resp} / \mathrm{min} \\
[\text { (IC } 95 \%) 24,87-25,52]\end{array}$} & $<0,001^{* *}$ \\
\hline Temperatura & \multicolumn{2}{|c|}{$\begin{array}{c}37,04+/-0,76 \text { OC } \\
{[(I C 95 \%) 36,98-37,10]}\end{array}$} & \multicolumn{2}{|c|}{$\begin{array}{c}37,12+/-0,77 \text { OC } \\
{[(\text { IC 95\%) } 37,08-37,16]}\end{array}$} & $0,036^{* *}$ \\
\hline $\begin{array}{l}\text { Saturación } \\
\text { Oxígeno }\end{array}$ & \multicolumn{2}{|c|}{$\begin{array}{c}77,34+/-13,27 \% \\
{[(I C 95 \%) 76,33-78,36]}\end{array}$} & \multicolumn{2}{|c|}{$\begin{array}{c}89,10+/-8,33 \% \\
{[\text { (IC 95\%) } 88,64-89,55]}\end{array}$} & $<0,001^{* *}$ \\
\hline $\begin{array}{l}\text { Estancia } \\
\text { hospitalaria }\end{array}$ & \multicolumn{2}{|c|}{$\begin{array}{c}\text { 7,42+/- 5,99 días } \\
{[(\text { IC } 95 \%) 6,96-7,88]}\end{array}$} & \multicolumn{2}{|c|}{$\begin{array}{c}\text { 10,61+/- 22,89 días } \\
\text { [(IC 95\%) 9,37-11,86 ] }\end{array}$} & $<0,001^{* *}$ \\
\hline
\end{tabular}

Tabla 3. Diabetes e hipertensión arterial como factores de riesgo en mortalidad en hospitalizados por COVID-19

\begin{tabular}{lcccc}
\hline \multirow{2}{*}{ Variable } & Fallecidos & Altas & \multirow{2}{*}{ OR } & (IC 95\%) \\
\cline { 2 - 3 } & $\%$ & $\%$ & & \\
\hline Diabetes & $17.00 \%$ & $13,1 \%$ & 1,4 & $(1,05-1,77)$ \\
$\quad \mathrm{Si}$ & $83.00 \%$ & $86,9 \%$ & & \\
$\quad$ No & & & & \\
Hipertensión arterial & $24.00 \%$ & $14,4 \%$ & 1,9 & $(1,49-2,39)$ \\
Si & $76.00 \%$ & $85,6 \%$ & & \\
No & & & & \\
\hline
\end{tabular}

relacionan con múltiples factores de confusión, la edad avanzada, consumo de tabaco y obesidad/sobrepeso, solo por mencionar algunas ${ }^{(7)}$. El mundo académico no ha podido demostrar fehacientemente la asociación entre la diabetes y la susceptibilidad al SARS Cov-2; no es claro el panorama de relación, la evidencia científica que se viene publicando demostraría que los pacientes con diabetes tienen mayor susceptibilidad, mayor riesgo; en los casos graves de enfermedad la 
prevalencia de diabetes oscila entre el $3 \%$ y $20 \%{ }^{(6,7)}$; las diferencias de resultados están asociadas al tamaño de la muestra, edad, gravedad de la enfermedad, nivel de complejidad del establecimiento sanitario, la presencia de otras comorbilidades, tratamiento previo al internamiento y tratamiento hospitalario, entre otras. En nuestro estudio la diabetes estuvo presente en el $14,4 \%$ del total pacientes adultos/adulto mayor, y en el $17,0 \%$ de los que fallecieron $(p=0,019)$.

La diabetes se encuentra entre las comorbilidades más prevalentes en pacientes adulto mayor, la diferencia en edad posiblemente está asociada al mayor o menor riesgo ${ }^{(8)}$. Se ha investigado el efecto de la diabetes en la progresión y mal pronóstico de los pacientes con COVID$19^{(9,10)}$, en los casos evaluados se demostró que los niveles séricos de IL-6, proteína $C$ reactiva y Dímero $D$ se encontraban en concentraciones mayores en pacientes con diabetes que en pacientes sin diabetes ${ }^{(6)}$, los biomarcadores indicarían un grave estado inflamatorio que podría estar asociado al mal pronóstico. El mal pronóstico podría estar relacionado con la propia inflamación, característica de la diabetes, la hipercoagulabilidad y la activación del sistema renina-angiotensina-aldosterona. En el presente estudio no se pudo conocer si los pacientes diabéticos estaban o no en tratamiento.

Hoy se conoce que el SARS CoV-2 usa los receptores ACE2 presente en las células epiteliales para poder ingresar en ellas11; ese tipo de receptores se localiza en tejido renal, cardiaco, pulmonar e intestinal. El sistema renina-angiotensina-aldosterona se encuentra modificado en los pacientes con diabetes ${ }^{(12)}$; la tormenta inflamatoria e hipercoagulable puede desencadenarse por cambios agudos en la actividad del ACE2. Algunos investigadores han propuesto que el uso de inhibidores del sistema renina-angiotensinaaldosterona mejora el pronóstico en el paciente diabético ${ }^{(13)}$

En nuestro estudio, la condición de paciente diabético representa 1.4 veces más riesgo de tener un desenlace fatal a la enfermedad.

Es conocido que las infecciones virales de vías respiratorias tienen mal pronóstico en pacientes con cáncer o inmunosupresión; se han reportado casos de daño y disfunción miocárdica en pacientes con COVID$19^{(14)}$. Estudios en Asia han determinado elevaciones de troponina y alteraciones en EKG en pacientes con COVID-19, la lesión cardiaca estaba presente en personas mayores y con HTA como comorbilidad ${ }^{(15)}$. La hipertensión es la comorbilidad y causa de muerte más común en pacientes con infección por COVID-19 ${ }^{(2)}$, las proteínas del SARS-CoV-2 tienen una alta afinidad de unión por ACE2, además, se ha demostrado que los inhibidores de la enzima convertidora de angiotensina (IECA) y los bloqueadores del receptor de angiotensina
(BRA) regulan al alza los niveles de ECA2, lo que media parcialmente sus efectos protectores cardiovasculares ${ }^{(16)}$. A la fecha, no se recomienda interrumpir tratamiento con IECA / ARA II, se puede provocar disfunción endotelial y, esta a su vez, provocar alteraciones en coagulación; la hipertensión podría conferir un estado protrombótico y sobreinflamación en pacientes con covid-19 $9^{(14-16)}$. Los resultados, en la población de estudio, determinaron que los pacientes con hipertensión arterial presentan 1,9 más riesgo de fallecer.

La diabetes y la hipertensión arterial están entre las comorbilidades que mayor riesgo implican para una presentación clínica grave en pacientes con la COVID19 , los hallazgos son de importancia para el adecuado abordaje terapéutico de los pacientes afectados y para el desarrollo de estrategias sanitarias en materia de prevención de complicaciones y tratamiento oportuno en el contexto de la pandemia.

Conflictos de interés: Los autores niegan conflictos de interés.

Financiamiento: Autofinanciado.

\section{REFERENCIAS BIBLIOGRÁFICAS}

1. Conflictos de interés: Los autores niegan conflictos de interés.

Financiamiento: Autofinanciado.

\section{REFERENCIAS BIBLIOGRÁFICAS}

1. Yang $X, Y u Y, X u$ J. Clinical course and outcomes of critically ill patients with SARS-CoV-2 pneumonia in Wuhan, China: a single-centered, retrospective, observational study Lancet Resp Med, F e bruary 24, 2020 D O I : https: / /doi.org/10.1016/S2213-2600(20)30079-5

2. Guan W, Ni Z, Hu Y. Clinical Characteristics of Coronavirus Disease 2019 in China. N Engl J Med. 2020 . D O I : https://doi.org/10.1056/NEJMoa2002032

3. Zhang JJ, Dong X, Cao YY. Clinical characteristics of 140 patients infected with SARSCOV-2 in Wuhan, China. Alergia. 2020. DOI: https://doi.org/10.1111/all.14238

4. Wan Y, Shang J, Graham R, Baric RS, Li F. Receptor Recognition by the Novel Coronavirus from Wuhan: an analysis based on decade-long structural studies of SARS Coronavirus. J Virology. 2020 DOI: https: / / doi.org/10.1128 / JVI.00127-20

5. Li XC, Zhang J, Zhuo JL. The vasoprotective axes of the renin-angiotensin system: Physiological relevance and therapeutic implications in cardiovascular, hypertensive and kidney diseases. Pharmacol Res 2017;125 (Pt A):21-38. DOI: 
https: / /doi.org/10.1016/j.phrs.2017.06.005

6. Guo W, Li M, Dong Y. Diabetes is a risk factor for the progression and prognosis of COVID-19. Diabetes Metab Res Rev. 2020; e 3319 . DOI: https://doi.org/10.1002/dmrr.3319

7. Wang X, Fang J, Zhu Y. Clinical characteristics of non-critically ill patients with novel coronavirus infection (COVID-19) in a Fangcang Hospital. Clin Microbiol I n fect. 2020 . DOI: https://doi.org/10.1016/j.cmi.2020.03.032

8. Lian J, Jin X, Hao S. Analysis of epidemiological and clinical features in older patients with Corona Virus Disease 2019 (COVID-19) out of Wuhan. Clin Infect Dis. 2020 ; ciaa242. DOI: https://doi.org/10.1093/cid/ciaa242

9. Shi S, Qin M, Shen B. Association of cardiac injury with mortal-ity in hospitalized patients with COVID-19 in Wuhan, China. JAMA Cardiol. 2020; $\begin{array}{lllllll}\text { e } 200950 \text {. D O I: } & \end{array}$ https://doi.org/10.1001/jamacardio.2020.0950

10. Guo T, Fan Y, Chen M, Wu X, Zhang L. Cardiovascular implications of fatal outcomes of patients with coronavirus disease 2019 (COVID19). JAMA Cardiol. 2020; e201017. DOI: https://doi.org/10.1001/jamacardio.2020.1017

11. Hoffman M, Kleine-Weber H, Schroeder S. SARSCoV-2 Cell Entry Depends on ACE2 and TMPRSS2 and Is Blocked by a Clinically Proven Protease Inhibitor. Cell. 2020; 181(2):271-280.e8. DOI: https://doi.org/10.1016/j.cell.2020.02.052

12. Patel VB, Parajuli N, Oudit GY. Role of angiotensinconverting en-zyme 2 (ACE2) in diabetic cardiovascular complications. Clin Sci (Lond). $2014 ; 126(7): 471-482$. D O I: https: / / doi.org/10.1042/CS20130344

13. Meng J, Xiao G, Zhang J. Renin-angiotensin system inhibitors improve the clinical outcomes of COVID-19 patients with hypertension. Emerg Microbes Infect. 2020; 9(1): 757- 760. DOI: https://doi.org/10.1080/22221751.2020.174620 $\underline{0}$

14. Kuster GM, Pfister O, Burkard T, Zhou Q, Twerenbold R, et al. SARS-CoV2: ¿should inhibitors of the renin-angiotensin system be withdrawn in patients with Covid-19? Eur Heart J. In press. DOI: https://doi.org/10.1093/eurheartj/ehaa235

15. Mehra MR, Desai SS, Kuy S, Henry TD, Patel AN. Cardiovascular disease, drug therapy, and mortality in Covid-19. New Engl J Med. In press. DOI: https://doi.org/10.1056/NEJMoa2007621

16. Sardu C, Maggi P, Messina V. ¿Podría la terapia con medicamentos antihipertensivos afectar el pronóstico clínico de los pacientes hipertensos con infección por COVID-19? Datos de centros del sur de Italia. J Am Heart Assoc. 2020; e016948. https://doi.org/10.1161/JAHA.120.016948.

\section{Correspondencia}

Dr. Pedro Javier Navarrete Mejía

Dirección: Av. Alameda del Corregidor 1517-1531, Urb. Sirius - III Etapa - La Molina.

Teléfono: 3(511) 365-2300 / (511) 365-3640

Correo: jnavarreteus@yahoo.com

\section{Revisión de pares}

Recibido: 22/10/2020

Aceptado: 15/12/2020 